\title{
Thermal Calibration of a 3 DOF Ultra High-Precision Robot Operating in Industrial Environment
}

\author{
Emanuele Lubrano and Professor Reymond Clavel, LSRO2 - EPFL - Switzerland
}

\begin{abstract}
-while dealing with sub-micrometer precision robots, thermal expansion is the most significant source of inaccuracy. Thermal variations in the environment, in the robot parts and in the frame change the robot geometry, lowering the robot precision. In this article we propose a strategy to model and compensate such effects. The thermal behavior of a 3 DOF (Degree(s)-of-freedom) parallel robot has been studied using a high-precision measuring system. A model of the robot thermal behavior has been built and implemented in the controller. By using it, thermal deformations are compensated in real-time and an absolute accuracy of $\pm 71 \mathrm{~nm}$ has been reached.
\end{abstract}

\section{INTRODUCTION}

$\mathrm{R}$ OBOT calibration consists in modeling and compensating the sources of inaccuracy that affect robot positioning [1]. Such causes of imprecision are considered according to the robot final application and the desired level of accuracy [2][3]. This article deals with the static calibration of a 3 DOF robot designed for submicrometer applications. In this case it is not sufficient to consider only the geometric errors of the robot: thermal variations play an important role in deforming robot parts.

In a previous work [4], the thermal compensation of a parallel linear axis has been carried out, achieving a final precision of $10 \mathrm{~nm}$. The procedure developed there has been adapted to our case, the calibration of the robot "Agietron Micro-Nano" (fig. 1 and 2). This robot has a delta parallel kinematic [5], 3 DOF in translations and the joint done by flexure hinges. Thanks to those features, the robot is fast, stiff and rigid, but with a small working-space. For those reason, the robot is currently used in industry to perform the EDM (electrical discharge machining) process [6]. The robot has a working space of $\sim 1 \mathrm{~cm}^{3}$ and a size of $\sim 20 \times 20 \times 25 \mathrm{~cm}$.

In this article we demonstrate the effectiveness of thermal calibration applied on ultra high-precision robots. Moreover, we analyze how temperature variations act on robot geometry and on the measuring loop.

Manuscript received September 15, 2009. This work was supported in part by the Swiss National Foundation for Research (FNS) and from EPFL.

Emanuele Lubrano is with the LSRO (Laboratory of Robotic Systems), EPFL, Lausanne, Switzerland (phone: +41 2169378 16; e-mail: emanuele.lubrano@epfl.ch).

Professor Reymond Clavel is with the LSRO, EPFL, Lausanne, Switzerland (e-mail: reymond.clavel@epfl.ch).

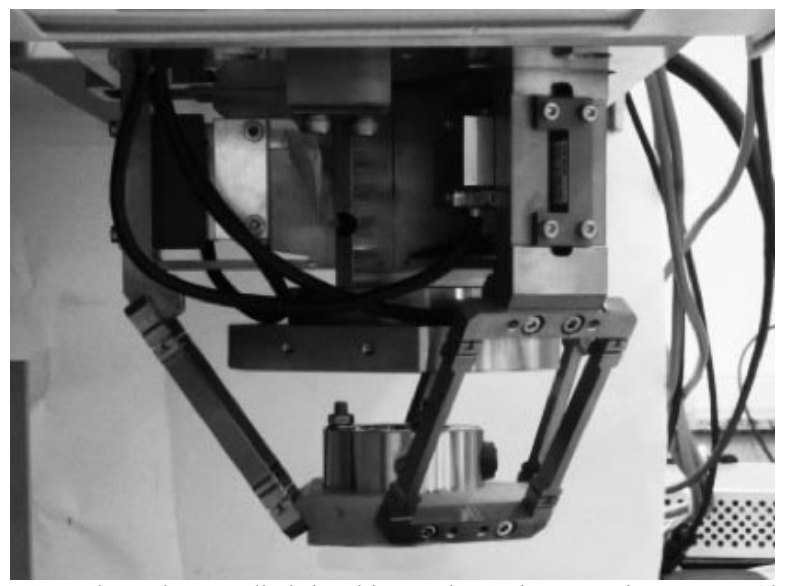

Fig. 1. The robot studied in this work, Agietron Micro-Nano (delta kinematic).

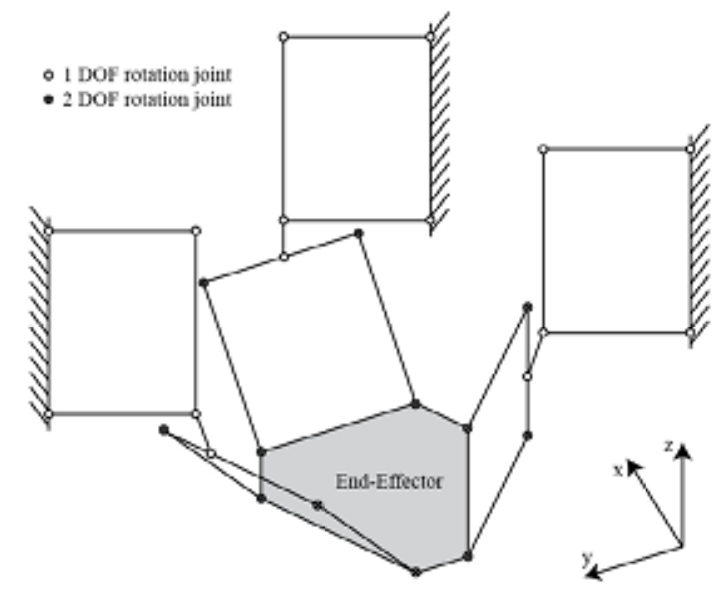

Fig. 2. Kinematic chain of the robot.

The whole work is divided in three parts: firstly we describe the development of the measuring system, then we show how we use it to acquire reliable measurements, and finally we show how we processed the data to perform the calibration.

\section{THE MEASURING SYSTEM}

\section{A. The measuring devices}

A 6 DOF measuring system has been conceived to measure translations, rotations and temperatures at very high-precision (fig. 3). 


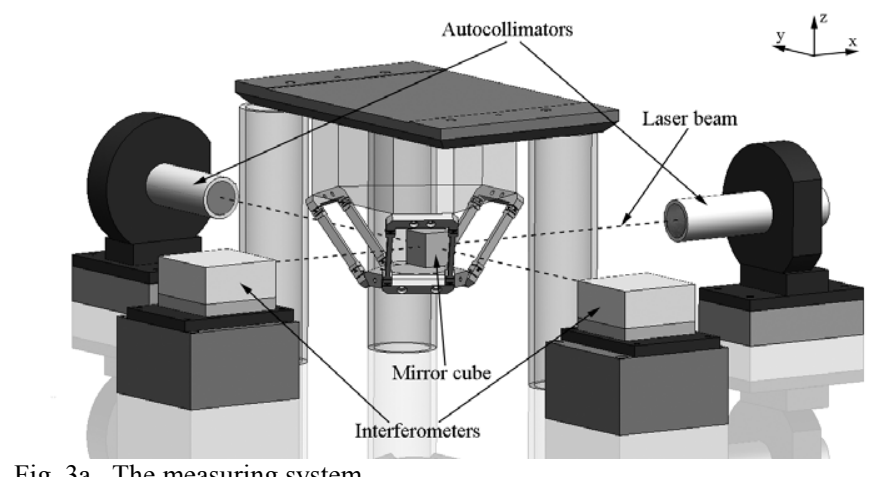

Fig. 3a. The measuring system.

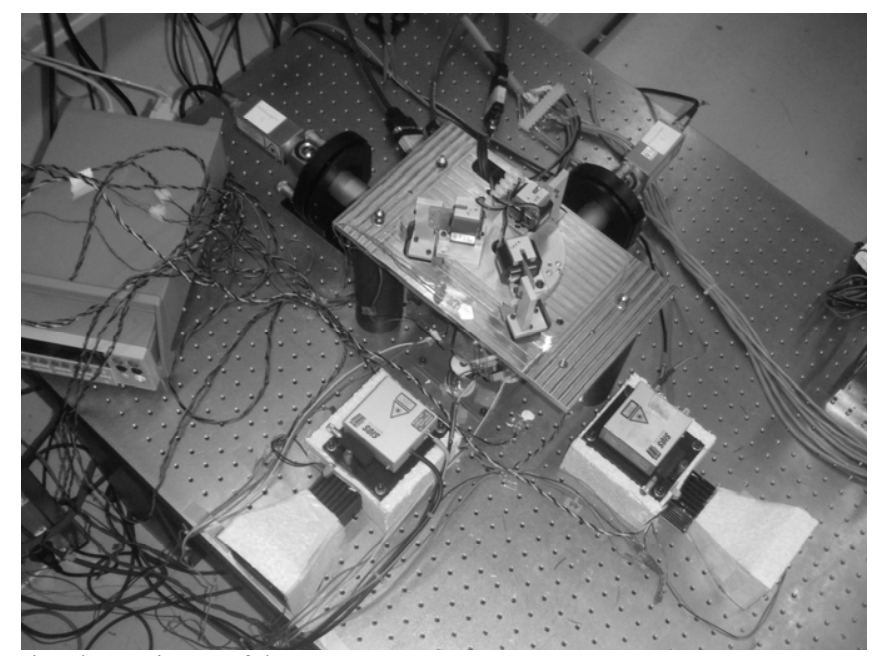

Fig. 3b. A picture of the system.

Translations are measured using 2 laser interferometers (SIOS SP-2000, resolution of $\sim 1.24 \mathrm{~nm}$, wavelength of $\sim 633$ $\mathrm{nm}$, stroke of $\sim 2 \mathrm{~m}$ ) mounted along the horizontal axes (X and $\mathrm{Y}$ ).

Rotations are measured using 2 autocollimators (Newport LDS-1000 Autocollimator, resolution of 0.02 arcsec, stroke of \pm 400 arcsec), capable of measuring 4 DOF (the vertical rotation axis is measured by both devices, allowing to do measuring confirmation tests). The principal aim of the rotation measurement is to compensate the end-effector parasitic rotations. In fact, those rotations affect the interferometer reading, adding the so called cosine error [7]. Errors dues to parasitic rotations are corrected in real-time.

Temperatures measurements are acquired using a total of 11 platinum resistance thermometers: 2 sensors measure the air temperature near the interferometers beams and 9 sensors are glued on the system, along the measuring loop and on the robot (fig. 4). The thermal measurements are acquired using a high-precision multi-channel A/D converter (Keithley 2700).

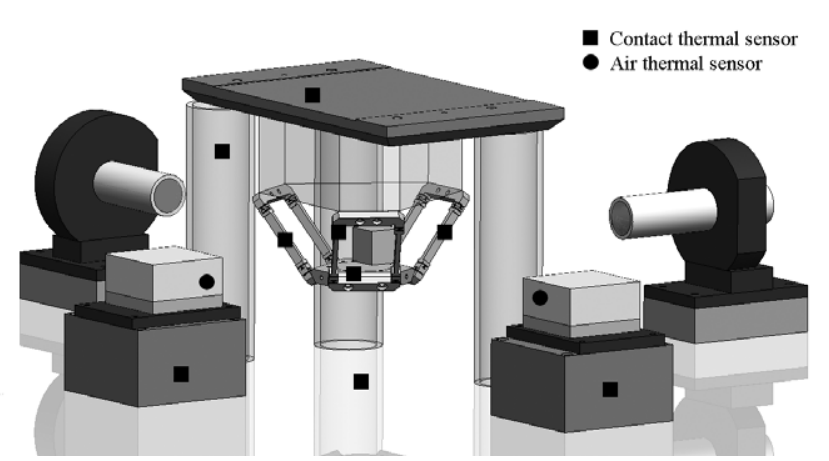

Fig. 4. Thermal sensors position.

Measurements are performed in two steps: during the first one, 2 interferometers and 2 autocollimators are used, acquiring a total number of 5 DOF; during the second, only one interferometer is used to measure the remaining vertical DOF. The vertical axis is measured using a $45^{\circ}$ mirrors mounted over the mirror cube, allowing mounting the interferometer in a horizontal position (compare with fig. 7).

\section{B. Considerations on thermal drift}

The measure of rotations is not affected by temperature drift [5]. On the contrary, translations must be measured with more attention. This because also the measuring devices are affected by thermal drift. Measurements collected neglecting the interferometer drift are wrong, because they do not represent the behavior of the measured item, but the behavior of the system "robot + measuring devices". Even if it is possible to find a mathematical model that fits such data, it will be false: in fact, manufacturing a piece with such a wrong model will not have the expected accuracy.

The thermal drift problem at nanometrical scale has been already solved in the past performing the thermal stabilization of the robot and the measuring system [8]. The lack of this procedure is the time: a thermal stabilization takes 8-10 hours to be performed, and such operation has to be done each time the robot is used. Consequently, this approach does not have any practical industrial application. In our study, thermal stabilization will be used only for the measuring equipments: thermal stabilization is not required during the normal use of the robot.

To limit the more as possible the drift of the interferometer, we use the air temperature compensation feature built-in the device. This will automatically compensate the drift due to internal components drift and the drift due to the changes of temperature in the air crossed by the laser beam. Secondarily, we stabilize the temperature of the interferometers supports. A Peltier cell is glued on each supports in order to control and stabilize it. A PID controller is used to command the Peltier cell, and the interferometer base is kept at the temperature of $25{ }^{\circ} \mathrm{C}$, with stability 
between $\pm 0.01{ }^{\circ} \mathrm{C}$ (fig. 5). To reach this level of stabilization only half an hour is needed. Two thermometers are used to control the Peltier cells. For this reason they will not be used for the thermal calibration, since they always show a value very near to $25^{\circ} \mathrm{C}$.

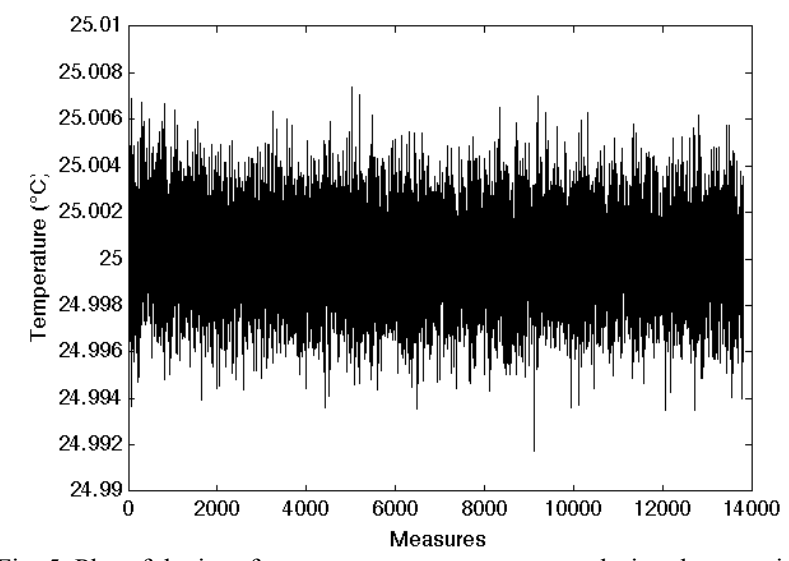

Fig. 5. Plot of the interferometer support temperature during the experiment.

Testing the system, we noticed that air flowing were responsible of augmenting the standard deviation of the measurements. We insulated the interferometers with a polystyrene box, then we covered the laser beam with an polystyrene tube and finally we covered all the system with a box (fig. 6 and 7). By using those devices we obtained a final standard deviation in the measurement of $5 \mathrm{~nm}$. This value has been recorded in all the working volume of the robot.

\section{Other parts of the system}

A mirror cube is mounted on the robot end-effector to reflect the laser beams of the measuring devices. It is built in Zerodur $^{\circledR}$, a material with an extremely low thermal expansion coefficient $\left(\sim 0.02 \times 10-6 / \mathrm{K}\right.$ at $\left.0-50^{\circ} \mathrm{C}\right)$. Moreover, it defines the origin and the frame of the system. The surface roughness of the cube is $30 \mathrm{~nm}$.

The origin or the "zero" of the measuring system in our case is reachable only at the beginning of the measuring session. Precisely, the zero is represented by 12 values: the $\mathrm{X}, \mathrm{Y}$ and $\mathrm{Z}$ measured by the interferometers (initialized to zero) and the 9 temperatures read by the sensors in the moment of the measure.

In Fig. 6 and 7 it is shown the path of the interferometers, the insulations and the different materials present in the system.

Notice also that all the system is mounted over a Newport vibration insulating table. The stabilization done on the interferometer bases affect the table temperature, heating it of 1-2 ${ }^{\circ} \mathrm{C}$, depending on environmental condition. It is important that also the table reach stability before starting the measurements. After that, the table and the robot follow the evolution of the temperature air. At last, robot motors and its support are not considerate in the stabilization because they are outside the measurement loop.

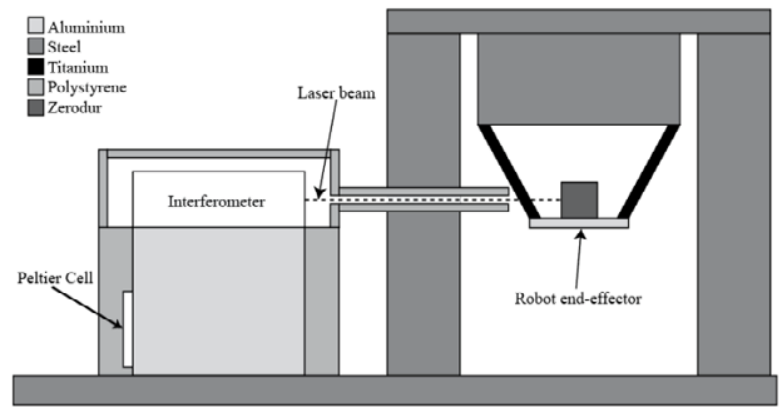

Fig. 6. Scheme of horizontal axis measurement.

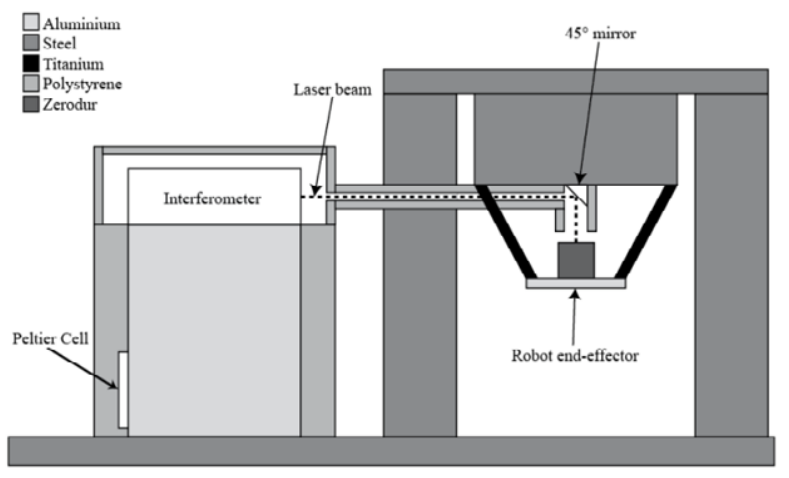

Fig. 7. Scheme of vertical axis measurement.

\section{MEASUREMENTS}

The measurements have been done during 3 days, while the room temperature was changing from $21{ }^{\circ} \mathrm{C}$ to $25^{\circ} \mathrm{C}$. We used the air conditioning (AC) to simulate the free oscillation of an industrial environment in the following way: before starting the measuring session, the AC consign has been putted to $20^{\circ} \mathrm{C}$. We started the measurements when the room reached the consign and during the measurements we turned off the air-conditioner. Therefore, the measurements have been collected while the temperatures were varying.

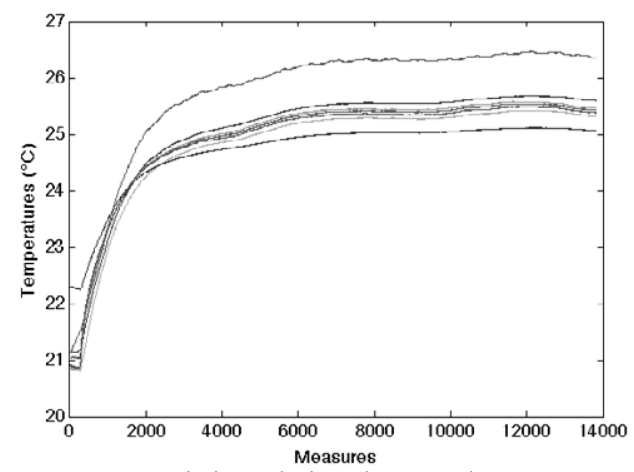

Fig. 8. Temperatures variations during the $\mathrm{X}$ and $\mathrm{Y}$ axes measurements, read on the sensors shown in Fig. 4. 
The data has been acquired during the weekend and all the equipment were controlled remotely. In this way the temperature drift due to operators entering in the robot's room has been minimized.

All the measuring instruments, the robot and the AC have been turned on before the measurements, in order to stabilize them. Furthermore, the robot was moving before the experiment, in order to heat also the motors. Depending on the initial condition, this warm-up phase can take between 1 and 3 hours.

When all the system is warmed-up, measurements are started. The $70 \%$ of the points acquired will be used to build a dataset called "calibration set", while the remaining 30\% will be used to validate the calibration (the "validation set"), The two sets contain different positions (so that the validation is not done in the points of calibration).

Acquiring one single measurement takes approximately 10 seconds. In this time the following sequence of actions is performed automatically:

- The robot moves to a new position.

- There is a waiting time of 5 seconds.

- The interferometers read continuously the displacements (one measurement is actually the filtered mean of 4096 measures, acquired in $0.2 \mathrm{~s}$ ). When the standard deviation of the measure fall under the $10 \mathrm{~nm}$, then a measure is acquired.

- The autocollimators measurements are acquired as well.

- The cosine effect is corrected.

- The last temperatures acquired are registered.

Temperatures are acquired in a separate loop, on a period of 10 seconds. It is not necessary to perform this operation faster because temperature changes are very slow.

\section{DATA PROCESSING}

The next step is to use the collected measurements to build a calibrated model of the robot. This model will have as input the desired end-effector position $(\mathrm{X}, \mathrm{Y}, \mathrm{Z})$ and the temperatures of the robot parts $\left(\mathrm{T}_{1}, \ldots, \mathrm{T}_{9}\right)$. As output, it will return the motor coordinates $\left(\mathrm{q}_{1}, \mathrm{q}_{2}, \mathrm{q}_{3}\right)$. In robotics, this is called "inverse geometric model" (eq. 1), IGM.

$$
q_{1}, q_{2}, q_{3}=f\left(X, Y, Z, T_{1}, \ldots, T_{11}\right)
$$

A model built in such way will keep in account the geometric features of the robot and its thermal behavior. This model will be done multiplying the variables seen before, and finding the good coefficients to fit the relation. To perform the coefficients research, we will use the "stepwise regression" algorithm (Matlab ${ }^{\circledR}$, Statistics Toolbox ${ }^{\mathrm{TM}}$ ). This algorithm has the capability of adding or removing terms from a multi-linear model. This is done comparing the statistical significance of the terms in a regression. The algorithm starts with an initial model that is compared with larger or smaller models. At each step, a coefficient is added to the model, thus, it is compared the final error with or without this last coefficient. If there is an improvement in the prediction, the coefficient is kept. Otherwise the coefficient is discarded. For the coefficients that are already in the model it happens the same: if the influence of any coefficient is under a certain threshold, the coefficient is rejected.

Depending on the terms included in the initial model and the order in which terms are moved in and out, the method may build different solutions from the same set of terms. The method terminates when any single step improves the model prediction capability. There is no guarantee that a different initial model or a different sequence of steps will not lead to a better fit. In this sense, stepwise models are locally optimal, but may not be globally optimal.

The stepwise regression algorithm has been chosen for two reasons: firstly it automatically deletes useless parameters, keeping the robot model computationally fast. Secondly, the algorithm converges and gives a solution in some seconds. On the contrary, algorithms tested in previous works (neural networks, gradient descent based parameters research, genetic algorithms and splines optimization) take some hours to give a solution.

\section{A. Generating the data for the calibration}

Since we can not read all the translations at the same time, we can not find directly the relationship (1). With the data available we can solve the inverse problem (eq. 2) for each axis singularly, the DGM (Direct Geometric Model). The solution of this problem will also show us the final accuracy of the robot after the calibration, in terms of articular coordinates.

$$
X, Y, Z=f\left(q_{1}, q_{2}, q_{3}, T_{1}, \ldots, T_{9}\right)(2)
$$

Excluding the temperatures, IGM is the inverted of DGM and vice-versa.

We can then split the calibration problem in 3 parts, calibrating each axis singularly:

$$
\left\{\begin{aligned}
X & =f\left(q_{1}, q_{2}, q_{3}, T_{1}, \ldots, T_{9}\right) \\
Y & =f\left(q_{1}, q_{2}, q_{3}, T_{1}, \ldots, T_{9}\right)(3) \\
Z^{*} & =f\left(q_{1}, q_{2}, q_{3}, T_{1}^{*}, \ldots, T_{9}^{*}\right)
\end{aligned}\right.
$$

Notice that we have used a star for the third equation. This because we had acquired the measures of the $\mathrm{Z}$ axis in another measuring session, so the temperatures were different from the first case.

We will now focus on how we calibrated one single axis; 
the procedure is the same for the remaining two.

What we want is a model that, given the motor coordinates, and the temperature values at a certain moment, it returns the interferometer reading along the $\mathrm{X}$ axis. For the moment we have only 12 variables to do so, we use these base variables to generate new ones: this is done by deriving it or by multiplying it, in order to see if the model fits the correlation of more variables.

From motors coordinates $\left(1^{\text {st }}\right.$ order $)$ we generate terms of the $2^{\text {nd }}$ and $3^{\text {rd }}$ order:

$$
\begin{aligned}
& 1^{\text {st }} \text { order: } q_{1}, q_{2}, q_{3} \\
& 2^{\text {nd }} \text { order: } q_{1}^{2}, q_{2}^{2}, q_{3}^{2}, q_{1} q_{2}, q_{2} q_{3}, q_{1} q_{3} \\
& 3^{\text {rd }} \text { order: } q_{1}^{3}, q_{2}^{3}, q_{3}^{3}, q_{1} q_{2} q_{3}, q_{1}^{2} q_{2}, q_{1}^{2} q_{3}, \\
& \qquad q_{2}^{2} q_{z 1}, q_{2}^{2} q_{3}, q_{3}^{2} q_{1}, q_{3}^{2} q_{2}
\end{aligned}
$$

From the departing 3 motors coordinates, we have generated 16 new correlation variables. In total, we have 19 pure geometrical variables.

Now we multiply those last 19 variables for the 9 temperatures reading that we have, and we obtain 171 correlated variables.

Thus, we calculate the first and the second derivative of each temperature, adding 18 new correlation variables to the 9 beginnings ones.

Adding all the variables together gives a final number of 217 variables. The calibration of one axis can be seen as the research of the coefficients $a_{1}, \ldots, a_{n}$ that satisfy the following relationship (eq. 4):

$$
\begin{aligned}
& {\left[\begin{array}{c}
X_{1} \\
\ldots \\
X_{m}
\end{array}\right]=A\left[\begin{array}{c}
a_{1} \\
\ldots \\
a_{n}
\end{array}\right]+b(4),} \\
& \mathrm{A}=\left[\begin{array}{cccccc}
q_{1,1} & \ldots & q_{1,1}^{2} & \ldots & T_{1,1} & \ldots \\
\ldots & & \ldots & & \ldots & \\
q_{1, m} & \ldots & q_{1, m}^{2} & \ldots & T_{1, m} & \ldots
\end{array}\right]
\end{aligned}
$$

where $\left[\begin{array}{lll}X_{1} & \ldots & X_{m}\end{array}\right]^{T}$ is the vector of the interferometer measurements along the $\mathrm{X}$ axis, $\mathrm{A}$ is an $m x n$ matrix containing the values of the motor coordinates and the value of the temperatures plus all the built coefficients corresponding to the interferometer measurement, $\left[\begin{array}{lll}a_{1} & \ldots & a_{m}\end{array}\right]^{T}$ is a vector containing the parameters that "stepwise regression" has to fit to make the (4) true and $b$ is an offset.

In the specific case of the $\mathrm{X}$ and $\mathrm{Y}$ axes calibration, $\mathrm{n}=$ 217 and $\mathrm{m}=9701$, while for the $Z^{*}$ case, $\mathrm{n}=217$ and $\mathrm{m}=$ 10390 .

Stepwise regression algorithm has been launched to solve this problem and only 74 parameters have been kept. The measurements in the calibration set have been fitted with an error of $\pm 59 \mathrm{~nm}$ in the $90 \%$ of the points.

Regarding the $\mathrm{Y}$ and the $\mathrm{Z}$ axes, we had respectively a model composed by 78 and 67 parameters, with an error in predicting the calibration set of \pm 54 and $\pm 94 \mathrm{~nm}$. We expected worst results in the vertical axis (the $\mathrm{Z}$ ), since the interferometer beam does a longer path, and it does 2 reflections over a mirror.

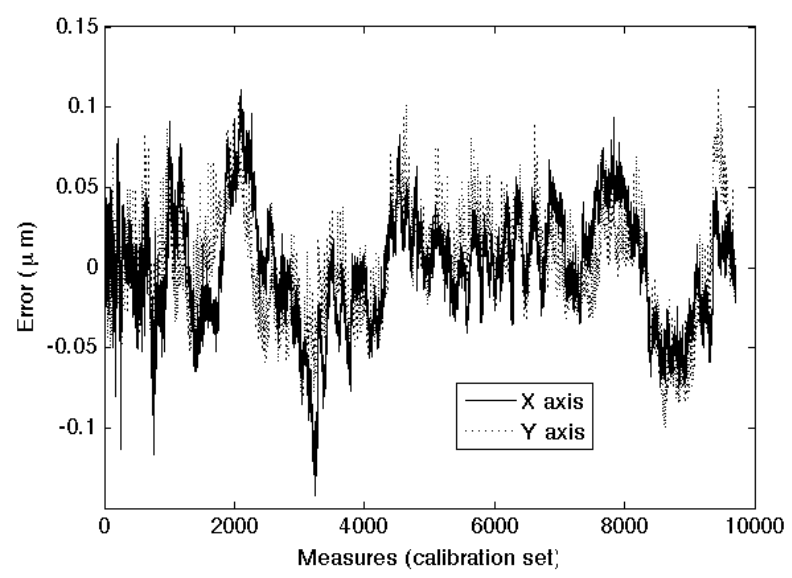

Fig. 9. The error in the calibration set.

\section{B. Obtaining the Inverse Geometric Model}

Since it is not possible to invert the coefficients matrix to find a numerical expression of the IGM, we will use a numerical technique that calls iteratively the DGM function until it founds the desired position. Specifically, we have used the Newton-Raphson method to implement the IGM in the robot controller.

\section{Results}

\section{A. Results in the prediction of the validation set}

To validate definitely the accuracy of the model, the validation set has been used. The validation set contains measures that have not been used to find the model coefficients. For the $\mathrm{X}$ and $\mathrm{Y}$ axes, the validation set is composed of 4074 measurements, while for the $\mathrm{Z}$ axis it is composed by 4327 measurements. The results in prediction are the following: an error of $\pm 96 \mathrm{~nm}$ for the $X$ axis, $\pm 71 \mathrm{~nm}$ for the $\mathrm{Y}$ axis and $\pm 150 \mathrm{~nm}$ for the $\mathrm{Z}$ axis for the $90 \%$ $(1.645 \sigma)$ of the validation set values. 


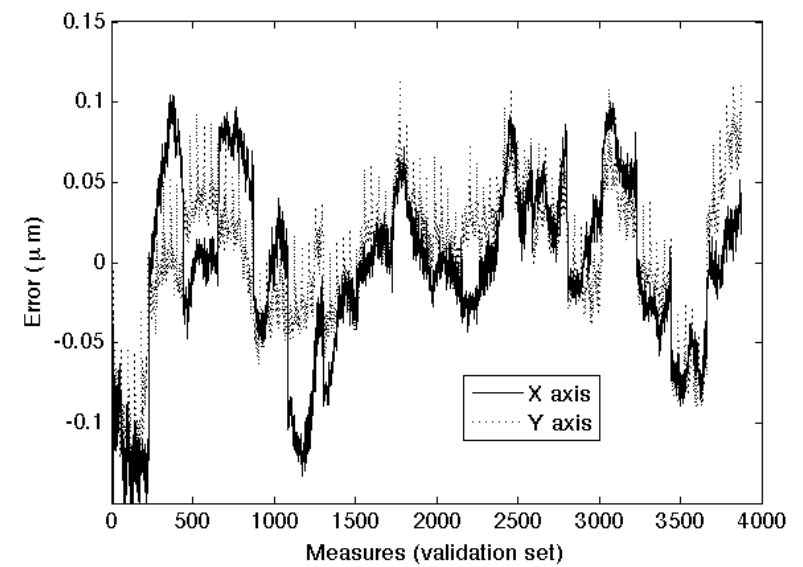

Fig. 10. The error in the prediction of the validation set.

\section{B. Influence of the parameters}

We finally performed some test to check the influence of the parameters in predicting the model.

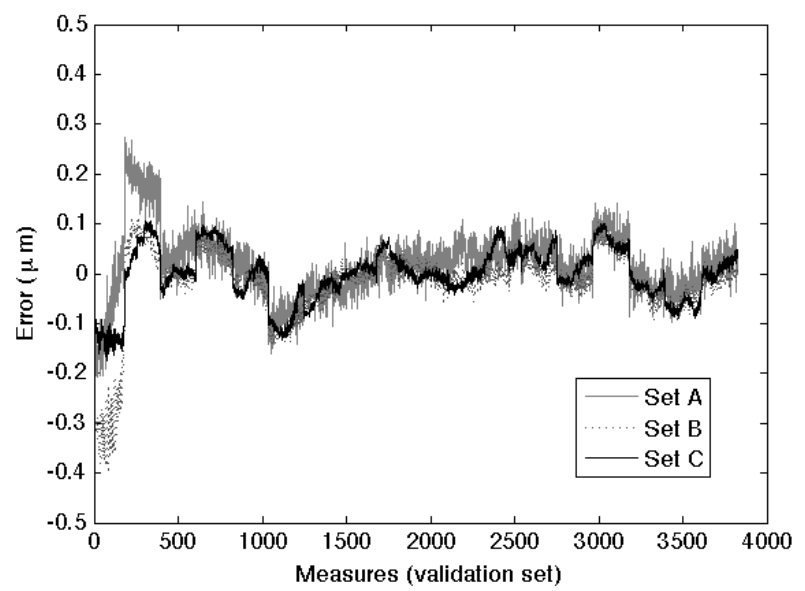

Fig. 11. Error using different sets of parameters along the $\mathrm{X}$ axis (Set $\mathrm{A}$ : geometric and pure thermal parameters; Set B: geometric, pure thermal and correlated thermal and geometric; Set C: complete set).

The results are shown only on the $\mathrm{X}$ axis, because they are similar in the other two cases (see fig. 11). Using only the geometric parameters, we have an error in the prediction of the validation set of $\pm 26,6 \mu \mathrm{m}$. Introducing the 9 reading of the temperature in this model will bring the error to \pm 115 $\mathrm{nm}$. Adding the temperatures correlated to the geometric parameters give $\pm 101 \mathrm{~nm}$, and - as we have already seen before - introducing the derivate of the temperatures brings the error to $\pm 96 \mathrm{~nm}$.

\section{CONCLUSION}

We have demonstrated that the thermal calibration procedure is effective also for a 3 DOF ultra high-precision robots. We seen also that a simple model composed only by geometrical and the pure thermal coefficients bring the final precision around $\pm 0.1 \mu \mathrm{m}$. Furthermore, we notice that correlated parameters and derivates increase the prediction, fine tuning the system.

In the future we will improve this work performing the following steps: firstly, we will develop a new 6 DOF measuring system, in order to acquire the measurements in only one step. Secondarily, we will study how cutting-forces influence the robot geometry and finally, we will consider a more complex case: a 2 ultra high-precision robots 6 DOF system.

The original contribution of this work are the development of a 6 DOF ultra high-precision measuring system, the development of an ultra high-precision calibration procedure that does not use a complete thermal stabilization, the calibration of a 3 DOF ultra high-precision robot in an unsteady industrial environment. All the procedure and the devices used in this work are suitable for industrial applications.

\section{REFERENCES}

[1] A.Y. Elatta. An overview of robot calibration. Whuhan, 2004.

[2] R. Ramesh et al. Error compensation in machine tools - a review. Part I: geometric, cutting-force induced and fixture-dependent errors. Singapore, Pergamon, 2000.

[3] R. Ramesh et al. Error compensation in machine tools - a review. Part II: thermal errors. Singapore, Pergamon, 2000.

[4] E. Lubrano et al. Thermal behavior of an ultra high-precision linear axis operating in industrial environment. Bergamo, 2008.

[5] R. Clavel. Conception d'un robot paralléle rapide à 4 degrés de liberté PhD Thesis n. 925, Lausanne, EPFL, 1991.

[6] C. Joseph. Contribution à l'accroissement des performances du processus de $\mu \mathrm{EDM}$ par l'utilisation d'un robot à dynamique élevée et de haute precision. PhD Thesis n. 3281, Lausanne, EPFL, 2005

[7] N. Fazenda. Calibration of high-precision flexure parallel robots. $\mathrm{PhD}$ Thesis n. 3712, Lausanne, EPFL, 2007.

[8] N. Fazenda et al. Calibration of the 6 DOF high-precision flexure parallel robot "Sigma 6". Chemnitz, Fraunhofer, 2006.

[9] W. Khalil et al. Modeling, identification and control of robots. Elsevier, 2004.

[10] Y. Da-Yong et al. Parallel robots pose accuracy compensation using artificial neural networks. Guangzhou, IEEE, 2005.

[11] R. Bernhardt. Robot calibration. Berlin, Chapman \& Hall, 1993.

[12] K.M. Lawton et al. A high-stability air temperature control system. Charlotte, Elsevier, 1999.

[13] D. Sarid et al. A \pm 15 microdegree temperature controller. American institute of Physics, 1974

[14] N. Srinivasa et al. Automated measurement and compensation of thermally induced error maps in machine tools. Precision engineering, Elsevier Science Inc., 1996.

[15] The MathWorks ${ }^{\mathrm{TM}}$ Statistics Toolbox ${ }^{\mathrm{TM}}$ 6, User guide. The MathWorks ${ }^{\mathrm{TM}}, 2008$. 